\title{
Physical exercise as chemosensitizer
}

\author{
Mauro Vaccarezza
}

Received: 22 August 2014/ Accepted: 1 September 2014/Published online: 9 September 2014

(C) Springer-Verlag Italia 2014

The benefits of physical exercise for the maintenance of human health, for the prevention and cure of metabolic syndrome and diabetes as well as for the prevention of cardiovascular diseases are well known [1]. Recently, a provocative study demonstrates an enhanced tumour perfusion and diminished tumour hypoxia in a model of orthotopic prostate cancer in rats exposed to treadmill exercise [2]. Of note, the measurements were performed on the conscious animal, which avoids the effects of anaesthetics on central haemodynamics and tumour blood flow, and provide an accurate evaluation of the resting versus exercising condition [2].

The reported blood flow increase of $200 \%$ and the $50 \%$ reduction in tumour hypoxia during exercise are unique findings [2] that are of particular interest to effectively enhance compound delivery to tumours. Intriguingly, a proposed mechanism is the "normalization" of the endothelial layer that is defective in tumours, and it represents a formidable barrier to efficient drug delivery [3, 4]. It would be interesting to assess the relative contribution of several types of exercise to these changes in endothelial permeability as well potential molecular mechanisms, keeping in mind a potential translation to these findings to clinical oncology. Of note, this provocative data are of interest even for blood cancers, considering the established role of bone marrow angiogenesis in the outcome of solid cancers and haematologic malignancies [3-5]. More experimental proof is definitely needed in animal models and in humans to support these results. Nevertheless, this is a possible inexpensive and valuable way to enhance drug potency [3, 4], with obvious translational effects in a clinical setting and possibly in cancer survival. Physical exercise science is coming of age not only in rehabilitation clinical haematology/oncology, but also as a potential therapeutic tool.

Conflict of interest None.

\section{References}

1. Vina J, Sanchis-Gomar F, Martinez-Bello V, Gomez-Cabrera MC. Exercise acts as a drug; the pharmacological benefits of exercise. Br J Pharmacol. 2012;167(1):1-12. doi:10.1111/j.1476-5381.2012. 0197022486393.

2. McCullough DJ, Stabley JN, Siemann DW, Behnke BJ. Modulation of blood flow, hypoxia, and vascular function in orthotopic prostate tumors during exercise. J Natl Cancer Inst. 2014; 106(4):dju036. doi: 10.1093/jnci/dju036. Epub 2014 Mar13.

3. Carmeliet P, Jain RK. Principles and mechanisms of vessel normalization for cancer and other angiogenic diseases. Nat Rev Drug Discov. 2011;10(6):417-27. doi:10.1038/nrd3455.

4. Jain RK. Normalizing tumor microenvironment to treat cancer: Bench to bedside to biomarkers. J Clin Oncol. 2013; 31(17):2205-18. doi:10.1200/JCO.2012.46.3653. Epub 2013 May 13.

5. Li WW, Hutnik M, Gehr G. Antiangiogenesis in haematological malignancies. Br J Haematol. 2008;143(5):622-31. doi:10.1111/j. 1365-2141.2008.07372.x.
M. Vaccarezza $(\bowtie)$

School of Biomedical Sciences, University of Queensland,

St.Lucia, Brisbane, QLD 4072, Australia

e-mail: m.vaccarezza@uq.edu.au

M. Vaccarezza

Department of Human, Social and Health Sciences, University of Cassino and Southern Lazio, 03043 Cassino, FR, Italy 